\title{
Frequency-Aware PPV: A Robust Phase Macromodel for Accurate Oscillator Noise Analysis
}

\author{
Xiaolue Lai \\ Cadence Design Systems, Inc. San Jose, CA \\ Email: laixl@cadence.com
}

\begin{abstract}
Perturbation Projection Vector (PPV) is an established technique for oscillator phase noise analysis; However, the PPV method significantly loses accuracy when circuits have large time constants, resulting in over-estimation of oscillator phase noise. In this paper, we show the problem of the PPV phase equation that it ignores the dynamics of oscillator frequency responses, and propose an improved PPV technique: Frequency-Aware PPV (FW-PPV). Using the FW-PPV technique, we derive analytical phase noise equations, which work accurately for all oscillators, especially for oscillators with large time constants. We apply the proposed technique on real oscillator circuits, and compare the results to Cadence Spectre RF. Simulation results show that our method has better accuracy than previous approaches.
\end{abstract}

\section{INTRODUCTION}

Oscillators are critical components in many electrical systems. In both RF and digital systems, accurate clock signals are required for correct operations. In oscillator based systems, the oscillator phase uncertainty has big impact to overall system performance, such as reducing timing margins in digital systems, and compromising channel separations in communications. The phase noise is the major concern, when designers design an oscillator circuit. As a result, accurate prediction of oscillator noise is one of the most important functionalities of nowadays simulation and verification tools.

The PPV [1] is a successful theory proposed for accurately characterizing oscillators' phase response to perturbations. PPV techniques have many applications: In [2] and [3], PPV phase macromodels have been proven to be able to simulate injection locking and PLL transient response much faster than full SPICE-level transient simulation, with small accuracy loss; In [1], authors derived analytical equations for oscillator noise prediction for white noise sources; in [4], the equation was extended to be applicable to both white and colored noise sources.

The phase noise prediction techniques in [1], [4] work well for small, fast oscillators, predicting the oscillator noise correctly. However, as techniques advance, designers tend to put more components (e.g., switch capacitors, DC bias, digital dividers, etc.) into oscillator cores. These extra components make the circuit larger and add large time constants, severely challenge all previous noise prediction methods. Large time constants significantly compromise the matrix conditioning of oscillators' linear periodic time-varying (LPTV) systems, making previous PPV extraction techniques [1], [4], [5] fail, or lose accuracy. In [6], a hierarchical approach was proposed for accurately extracting the PPV waveforms of oscillators with large time constants. However, although the PPV can be extracted correctly, the oscillator noise analysis is still inaccurate: the phase noise is always over-estimated.

In this work, we investigate the root reason why PPV techniques over-estimate phase noise when oscillators have large time constants. In PPV techniques, a unified phase equation and PPV waveform are used to capture the oscillator phase responses under any perturbations, regardless of perturbation frequencies. In the PPV phase equation, the dynamics of oscillator frequency responses is ignored: the impulse response of oscillator frequency deviation is approximated using series of pulses whose magnitude is the PPV. The approximation could be problematic, especially for slow oscillators, because oscillator frequency, like voltages and currents in a circuit, cannot change infinitely fast - a physical system has limited bandwidth. The phase equation was verified to be correct in many previous works [1], [2], because it was evaluated on small, simple oscillators, and perturbations were applied to oscillator cores. Since oscillator cores have very wide bandwidth, ignoring the dynamics may not compromise the macromodelling accuracy very much. However, in oscillator noise analysis, we need to calculate the total noise contributed by all devices in the circuit. Slow nodes in the oscillator invalidate the PPV phase equation, because the dynamics of frequency responses cannot be ignored: large time constants filter out the noise from the nearby devices and reduce the total noise. As a result, the PPV technique always over-estimates oscillator total noise because it is not able to capture the filtering effects.

To improve the phase noise analysis accuracy, we need to consider the PPV as a frequency-dependent quantity - different perturbation frequencies should have different PPV waveforms. In this work, we present the Frequency-Aware PPV (FW-PPV) technique. In our approach, we calculate the PPV for different small signal frequencies and construct time domain impulse response waveforms. Using the impulse response waveforms, instead of the pulse, in the phase equation, the FW-PPV predicts oscillator phase responses more accurate than previous PPV techniques. In [7], an oscillator AC analysis method has been presented: Oscillator frequency is treated as a state variable, like node voltages, and then the periodic AC analysis (PAC) can be applied to calculate the frequency deviation of oscillators under periodic perturbations. We show that the FW-PPV can be extracted by solving the adjoint system of the oscillator AC analysis - the FW-PPV is a periodic transfer function (PXF) analysis for the oscillators' frequency. We also show that the PPV is a special case of the FW-PPV, solved at the small signal frequency of $0 \mathrm{~Hz}$.

Using the FW-PPV technique, we derive new analytical equations for oscillator noise prediction. The resulting noise analysis technique is applicable to any oscillator, especially for oscillators with large time constants - the phase noise results are not over-estimated. The FW-PPV technique has two major advantages: Firstly, it is more accurate when applying to real oscillators. Secondly, the matrix conditioning is improved for ill-conditioned oscillators, when the small signal frequency is incorporated. We do not need to implement complicated PPV extraction techniques for ill-conditioned oscillators (e.g., the hierarchical technique [6]) - a plain periodic transfer function analysis code can do the PPV extraction.

We implement the proposed FW-PPV technique, and compare it to Cadence Spectre RF, which uses the PPV technique. The results are as expected: When oscillators have no large time constant, the FWPPV technique works equally well, giving exactly the same phase noise plot as Spectre RF; However, when oscillator has large time constants, Spectre $R F$ loses accuracy, giving over-estimated phase noise; On the contrary, the FW-PPV is not affected by the large time constants - the phase noise plot from the FW-PPV can be proven to be correct.

The remainder of the paper is organized as follows: In Section II, we briefly review the ppv technique, and show its problem when circuits have large time constants. In Section III, we present the FW-PPV technique. In Section IV, we evaluate the FW-PPV on real oscillators by comparing to Spectre $R F$. 


\section{PPV Macromodel AND Oscillator Phase Noise ANALYSIS}

In this section, we first provide a brief overview of the PPV macromodel [1] and according oscillator phase noise analysis techniques [1], [4]. Then we show the shortcoming of the PPV when circuits have large time constants.

\section{A. PPV Technique}

A perturbed oscillator can be expressed as a DAE equation

$$
\dot{q}(x(t))+f(x(t))=b(t),
$$

where $b(t)$ is the perturbation applied to the free-running oscillator. The solution of the perturbed oscillator can be shown to be of the form [1]

$$
x_{p}(t)=x^{*}(t+\alpha(t))+y(t),
$$

where $x_{p}(t)$ is the perturbed solution, $x^{*}(t)$ is the steady-state solution, $\alpha(t)$ is the phase shift due to perturbation $b(t)$, and $y(t)$ is the amplitude variations.

Using (2), the total deviation $x(t)$ can be expressed as

$$
x(t)=p(t)+y(t),
$$

where $p(t)=x^{*}(t+\alpha(t))-x^{*}(t)$ is the orbit deviation due to phase shift $\alpha(t)$, which is governed by a scalar differential equation [1]:

$$
\dot{\alpha}(t)=v_{1}^{T}(t+\alpha(t)) \cdot b(t),
$$

where $v_{1}(t)$ is the PPV, a vector of periodic waveforms with the same period as that of the free-running oscillator.

In [1], $p(t)$ is considered to be the major contributor of the oscillator noise and the amplitude variations $y(t)$ can be ignored. As a result, oscillator noise can be approximated using

$$
x(t)=p(t)=x^{*}(t+\alpha(t))-x^{*}(t) .
$$

Since $p(t)$ can be easy calculated after $\alpha(t)$ is solved using (4), the PPV technique offers an efficient way for predicting oscillator noise.

\section{B. PPV Extraction Method}

There are different numerical methods for calculating the PPV. Among them, the harmonic balance augmented Jacobian method has the best efficiency and robustness.

For oscillators, the frequency is an extra unknown in the harmonic balance simulation. When harmonic balance converges, the Jacobian matrix, which is called augmented Jacobian matrix, has the form of

$$
J_{\text {aug }}=\left(\begin{array}{cc}
J & q \\
p^{T} & r
\end{array}\right)
$$

where $J$ is the harmonic balance Jacobian of the oscillator equation, $p$ is the vector of phase condition, $q=\Omega Q$ is the Fourier coefficient of $\dot{q}\left(x^{*}(t)\right)$, and $r$ is a scalar.

If the augmented Jacobian matrix is not singular, the PPV can be calculated by solving a linear equation

$$
J_{\text {aug }}^{T} X=e
$$

where $e=[0,0, \ldots, 0,1]^{T}$, and $X=\left[V_{1}, d\right]^{T}$ in which $V_{1}$ is the Fourier coefficients of the PPV, and $d$ is a scalar.

\section{Oscillator Phase Noise Prediction}

In [1], authors derived an analytical equation for calculating oscillator phase noise for white noise sources using the PPV. The equation is

$$
S(f)=\sum_{i=-N}^{N} X_{i} X_{i}^{*} \frac{f_{0}^{2} i^{2} c_{w}}{\pi^{2} f_{0}^{4} i^{4} c_{w}^{2}+\left(f+i f_{0}\right)^{2}},
$$

where

$$
c_{w}=\frac{1}{T} \int_{0}^{T} v_{1}^{T}(t) v_{1}(t) d t
$$

In this equation, $v_{1}(t)$ is the PPV, $X_{i}$ is the Fourier coefficient of the oscillator output at harminic index $i, f_{0}$ is the fundamental frequency, and $f$ is the small signal frequency. To use this equation, all noise sources need to be white noise with power of 1 .

In [4], authors derive another analytical equation for colored noise source. The equation has the form

$$
S(f)=\sum_{i=-N}^{N} X_{i} X_{i}^{*} S_{i}\left(f+i f_{0}\right),
$$

where

$$
S_{i}(f)=\frac{f_{0}^{2} i^{2} \sum_{k=1}^{n}\left|V_{0_{k}}\right|^{2} S_{N_{k}}(f)}{\pi^{2} f_{0}^{4} i^{4}\left(\sum_{k=1}^{n}\left|V_{0_{k}}\right|^{2} S_{N_{k}}(f)\right)^{2}+f^{2}} .
$$

In this equation, $V_{0_{k}}$ is the DC Fourier coefficient of the PPV of the node $k$, and $S_{N_{k}}$ is the PSD of all colored noise sources applied to the node $k$.

If circuits have both white and colored noise source, (11) can be written as

$$
S_{i}(f)=\frac{f_{0}^{2} i^{2}\left(\sum_{k=1}^{n}\left|V_{0_{k}}\right|^{2} S_{N_{k}}(f)+c_{w}\right)}{\pi^{2} f_{0}^{4} i^{4}\left(\sum_{k=1}^{n}\left|V_{0_{k}}\right|^{2} S_{N_{k}}(f)+c_{w}\right)^{2}+f^{2}},
$$

where $c_{w}$ is defined in (9)

(12) works well on small and fast oscillators; however, when oscillators have many slow time constants, (12) may over-estimate the phase noise, as shown in Section IV.

\section{Problem of the PPV Macromodel}

To show the problem of the PPV macromodel, we rewrite the phase equation (4) by using the frequency deviation as state variable. In (4), $\alpha(t)$ is the phase deviation, which satisfies

$$
\dot{\alpha}(t)=\Delta \omega(t),
$$

where $\Delta \omega$ is the frequency deviation of the oscillator.

Substituting (13) into (4), we have

$$
\Delta \omega(t)=v_{1}^{T}(\hat{t}) \cdot b(t)
$$

where $\hat{t}=t+\alpha(t)$.

Since small signal responses of oscillators are linear periodic timevarying systems, the frequency deviation of an oscillator should have a convolution form:

$$
\Delta \omega(t)=\int_{0}^{t} h_{\omega}^{T}(\hat{\tau}, \hat{t}) \cdot b(\tau) d \tau,
$$

where $h_{\omega}^{T}(\hat{\tau}, \hat{t})$ is the impulse response of the oscillator frequency.

Comparing (14) with (15), it is obvious that the dynamics of the oscillator frequency response is ignored in the phase equation: The impulse response of the oscillator frequency deviation is approximated using a pulse with the magnitude of $v_{1}^{T}(\hat{t})$. The approximation is only accurate when the oscillator circuit has no slow constant. As a result, the PPV method lose accuracy when oscillators have large time constants.

We can also explain the problem of the PPV in a graphical way. According to equation (3), the total deviation $x(t)$ can be decomposed into two parts: $p(t)$, the orbit deviation due to phase shift and $y(t)$, the amplitude variations. For an oscillator without large time constant, this decomposition can be shown as Figure 1(a). $p(t)$ dominates in the plot, hence, it is valid to use $p(t)$ to approximate the total noise $x(t)$. However, when the oscillator has large time constants, the Jacobian matrix is close to singular, and the PPV vector has some very large entries. As a result, the total deviation $x(t)$ is decomposed into two very large components, as shown in Figure 1(b). When this happens, using the PPV will over-estimate the oscillator noise.

\section{FREQUENCY-AwARE PPV TEChNIQUE}

In this section, we first derive a general phase equation, which takes the perturbation frequency into consideration. Then we present 


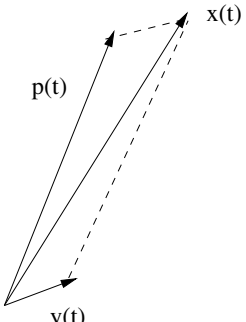

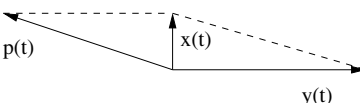

$\mathrm{y}(\mathrm{t})$ (a) Oscillator without (b) Oscillator with large time large time constant. constant.

Fig. 1. Decomposition of oscillator total deviation.

method for calculate the frequency-aware PPV. In the end, we present FW-PPV based analytical equations for predicting oscillator phase noise.

\section{A. Frequency-Aware Oscillator Phase Equation}

Since (4) has problem when nodes have large time constants. We need to re-derive a more general phase equation.

We know the relation between phase deviation and frequency deviation can be expressed by an implicit differential equation [7]:

$$
\dot{\alpha}(t)=\Delta \bar{\omega}(t+\alpha(t)),
$$

where $\Delta \bar{\omega}=\frac{\Delta \omega}{\omega_{0}}$, is the normalized frequency deviation. If we have a way to get $\Delta \bar{\omega}$, we can calculate phase deviation by solving (16).

Oscillators are special circuits: their frequencies are treated as a state variable, like node voltages, in simulations. Oscillator frequency changes when perturbations are applied. Hence, there should have impulse responses of oscillator frequency deviation to perturbations. Since the linearized oscillator system is an LPTV system, the impulse responses should have the form

$$
h_{\Delta \bar{\omega}}(\tau, t),
$$

which gives us the frequency deviation at time $t$ after an impulse is applied at time $\tau$.

Assume we have the frequency deviation impulse response $h_{\Delta \bar{\omega}}(\tau, t)$ (we will discuss how to calculate it in next section), the oscillator frequency deviation can be calculated by doing the convolution

$$
\Delta \bar{\omega}(t)=\int_{0}^{t} h_{\Delta \bar{\omega}}^{T}(\tau, t) \cdot b(\tau) d \tau,
$$

where $h_{\Delta \bar{\omega}}$ is a vector, in which each entry is an impulse response of frequency deviation to one input.

Substituting (18) into (16), we obtain a general phase equation for oscillator phase deviation:

$$
\dot{\alpha}(t)=\int_{0}^{(t+\alpha(t))} h_{\Delta \bar{\omega}}^{T}(\tau+\alpha(\tau), t+\alpha(t)) \cdot b(\tau) d \tau,
$$

If we assume the oscillator's frequency response is infinitely fast, or

$$
h_{\Delta \bar{\omega}}(\tau, t)=v_{1}(t) \delta(t-\tau) .
$$

Substituting (20) into (19), we obtain

$$
\dot{\alpha}(t)=v_{1}^{T}(t+\alpha(t)) \cdot b(t),
$$

the PPV phase equation. Hence, the PPV phase equation is a special case of the FW-PPV phase equation, which ignores the dynamics of oscillator frequency responses.

\section{B. FW-PPV Extraction}

In this section, we present a method to extract $h_{\Delta \bar{\omega}}(\tau, t)$ from the oscillator's LPTV system.
In [7], the oscillator frequency is treated as a state variable, and oscillator PAC analysis can be done by solving

$$
\left(j \omega_{s}\left[\begin{array}{ll}
C & 0 \\
0 & 0
\end{array}\right]+\left[\begin{array}{lr}
J & \mathbb{T}_{\Omega Q} \\
\Omega \mathbb{T}_{e_{l}^{T}} & \mathbb{T}_{\dot{x}_{l}^{*}}
\end{array}\right]\right)\left(\begin{array}{l}
\Delta X\left(\omega_{s}\right) \\
\Delta \bar{W}\left(\omega_{s}\right)
\end{array}\right)=B .
$$

In this equation, $J=\Omega C+G$, is the Jacobian matrix of the oscillator equation, $C$ is the circulant matrix of the dynamic part of the circuit equation, and $\omega_{s}$ is the small signal frequency. $\mathbb{T}_{\Omega O}$ is a Toeplitz matrix that circulates $\Omega Q$, the Fourier coefficient of $\dot{q}\left(x^{*}(t)\right)$, and $\Omega \mathbb{T}_{e_{l}^{T}}$ and $\mathbb{T}_{\dot{x}_{l}^{*}}$ are phase equations to make the system to be square [7].

$\Delta W\left(\omega_{s}\right)$ in this equation gives us the frequency deviation under perturbation $B$ with perturbation frequency of $\omega_{s}$. Solving (22) gives us the transfer function from one of circuit node to oscillator's frequency deviation. If we want transfer functions from all nodes, we need to do a PXF by solving the adjoint system

$$
\left(j \omega_{s}\left[\begin{array}{ll}
C & 0 \\
0 & 0
\end{array}\right]+\left[\begin{array}{ll}
J & \mathbb{T}_{\Omega Q} \\
\Omega \mathbb{T}_{e_{l}^{T}} & \mathbb{T}_{\dot{x}_{i}^{*}}
\end{array}\right]\right)^{T}\left(\begin{array}{l}
H_{\Delta \bar{\omega}}\left(\omega_{s}\right) \\
P
\end{array}\right)=\left(\begin{array}{l}
0 \\
C
\end{array}\right),
$$

where $C=[0, \ldots, 0,1,0, \ldots, 0]^{T}$, and $H_{\Delta \bar{\omega}}$ gives us transfer functions of frequency deviation for all input nodes at perturbation frequency $\omega_{s}$. We can sweep $\omega_{s}$ to calculate the transfer functions at different small frequencies and do a 2D inverse Fourier transform to get $h_{\Delta \bar{\omega}}(\tau, t)$ for the FW-PPV phase equation.

(23) is very difficult to solve using iterative solvers (such as GMRES [8]), because the extra columns and rows from $\mathbb{T}_{\Omega Q}$ and $\Omega \mathbb{T}_{e^{T}}$ degrade the block diagonal preconditioner, making GMRES difficult to converge. $\mathbb{T}_{\Omega Q}$ has $N$ columns, where $N$ is the total harmonic number. if we are only interested in the transfer functions when $\omega_{s}$ is close to DC (this is the case for phase noise analysis), we can drop all AC columns in $\mathbb{T}_{\Omega Q}$ and only keep the middle column, which is $\Omega Q$, resulting in a simplified equation:

$$
\left(j \omega_{s}\left[\begin{array}{ll}
C & 0 \\
0 & 0
\end{array}\right]+\left[\begin{array}{lr}
J & \Omega Q \\
p^{T} & r
\end{array}\right]\right)^{T}\left(\begin{array}{l}
H_{\Delta \bar{\omega}}\left(\omega_{s}\right) \\
P
\end{array}\right)=\left(\begin{array}{l}
0 \\
1
\end{array}\right),
$$

where $\Omega Q$ and $p^{T}$ are vectors, and $p^{T}$ is a single phase equation. (24) looks very familiar: if we make the small frequency $\omega_{s}=0$, it is the augmented PPV extraction equation: (6) and (7) in Section II-B. It is clear now that the previous PPV extraction methods give us the transfer function at $\omega_{s}=0$.

\section{Phase Noise Prediction Using FW-PPV}

Follow the procedure in [1], [4], and use the FW-PPV phase equation (19), instead of (4), we can derive a new analytical equation for oscillator phase noise analysis:

$$
S(f)=\sum_{i=-N}^{N} X_{i} X_{i}^{*} \frac{f_{0}^{2} i^{2} \cdot c\left(f+i f_{0}\right)}{\pi^{2} f_{0}^{4} i^{4} \cdot c\left(f+i f_{0}\right)^{2}+\left(f+i f_{0}\right)^{2}},
$$

where $c(f)$ is a frequency dependent scalar function defined by

$$
c(f)=\sum_{i=1}^{n} \sum_{k=-N}^{N}\left|H_{\Delta \bar{\omega}_{i, k}}(f)\right|^{2} S_{n_{i}}\left(-k f_{0}+f\right),
$$

In this equation, $n$ is circuit size, $N$ is harmonic number, $i$ is node index, $k$ is harmonic index, $H_{\Delta \bar{\omega}_{i, k}}$ is the frequency deviation transfer function of node $i$ on harmonic $k$, and $S_{n_{i}}$ is the noise PSD of all noise applied to node $i$. Once we extract $H_{\Delta \bar{\omega}_{i k}}$ using the method from the previous section, we can predict the oscillator phase noise using (25) and (26).

\section{Simulation Results}

In this section, we evaluate the proposed FW-PPV technique. We implement the FW-PPV technique, and compare results to Cadence Spectre $R F$, which uses the PPV technique. The results from Spectre 
$R F$ and our method are written to ASCII files and post-precessed using MATLAB. Simulation results show that our approach has better accuracy than the PPV technique: When oscillators have no large time constants, our method gives exactly the same results as Spectre $R F$. In addition, our method can plot the Lorentzian corner frequency correctly, while Spectre $R F$ cannot plot the Lorentzian it outputs corner frequency in simulation summary; When oscillators have large time constants, Spectre $R F$ over-estimates the oscillator phase noise, giving very large phase noise outputs and unreasonable corner frequencies; On the contrary, the FW-PPV method is not affected by large time constants: its phase noise results are reasonable and as we expect.

In the first example, we simulate the phase noise of a BJT LC oscillator. The circuit uses 146 BJT devices, and the total equation number is 941 . The output of the oscillator has the magnitude of about $0.5 \mathrm{~V}$, and the oscillator has the oscillating frequency of $1.83 \mathrm{GHz}$. Since this circuit is well-conditioned (there has no large time constant in the circuit), the FW-PPV works equally well as Spectre $R f$ for the phase noise prediction. The simulation results are shown in Figure 2. The corner frequency in the FW-PPV plot is $24.6 \mathrm{KHz}$, which is exactly the same number from Spectre $R F$ simulation summary. Two waveforms have very good match when the offset frequency is larger than the corner frequency. This simulation confirms that the FW-PPV and the PPV work equally well for normal oscillators without large time constants.

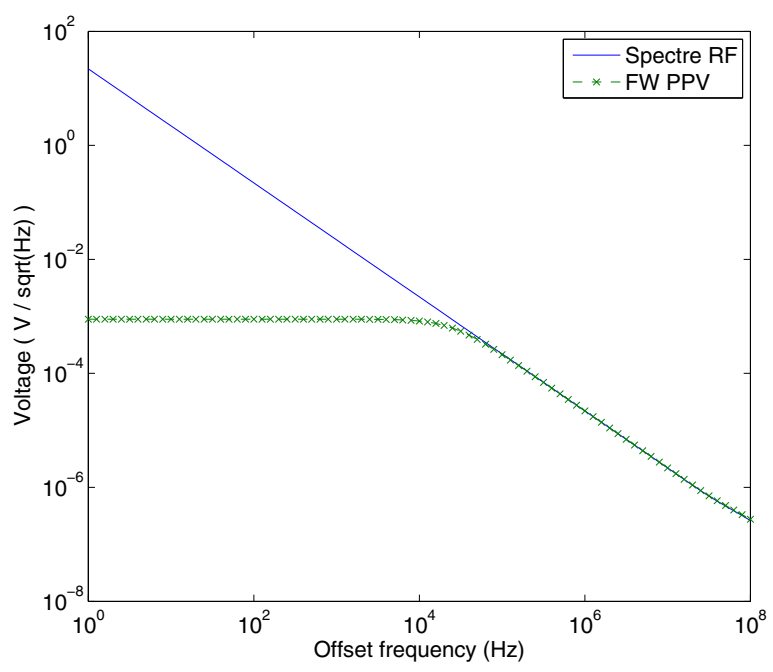

Fig. 2. Phase noise output of the BJT LC oscillator.

In the second example, we try both methods on a gated capacitor tuning LC oscillator. The circuit has 226 MOSFETs, and the total equation number is 813 . The output of the oscillator has magnitude $2.5 \mathrm{~V}$ and frequency $3.15 \mathrm{GHz}$. Since some of the gated capacitors are turned off, the circuit has very large time constants.

We simulate the phase noise of the oscillator using both methods, and show the results in Figure 3. In this figure, we plot three phase noise waveforms. The solid line is the reference. To get the reference waveform, we disable the noise contribution of gates that are turned off in the netlist, and simulate the phase noise using Spectre $R F$. Since the RC time constants of the "off" gated capacitors is very large (larger than 1), it is safe to assume that these gates have very small contribution to the total phase noise when offset frequency is reasonably large. Hence, it is valid to use this waveform as the reference to benchmark our methods. It can be easily seen from Figure 3 that the result from the FW-PPV method has very good match to the reference; however, the PPV method gives us an overestimated phase noise output (the green dash line): it is about $20 \mathrm{db}$ higher in the worst case. The corner frequency from Spectre $R f$ is about $1.26 \mathrm{MHz}$. This is also unreasonable, because an well-designed LC oscillator cannot have such large phase noise to spread power to more than $1 \mathrm{MHz}$.

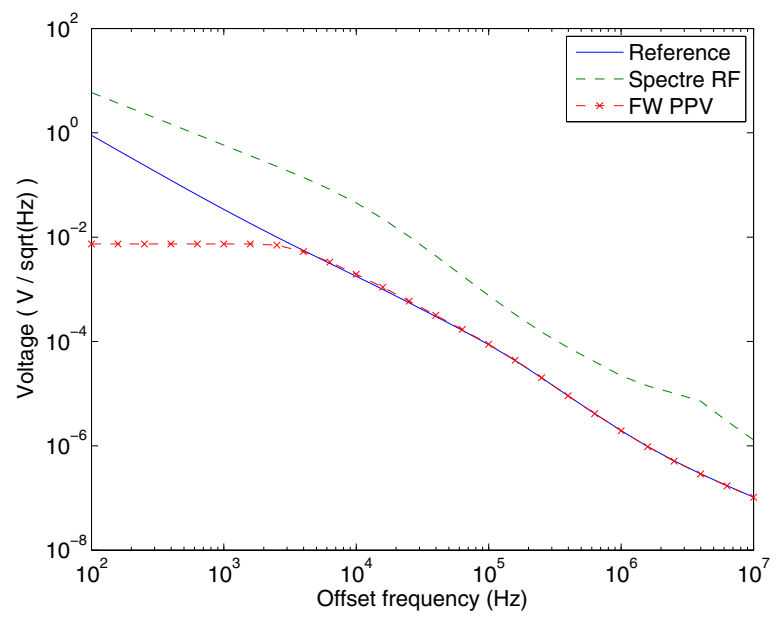

Fig. 3. Phase noise output of the gated capacitor tuning LC oscillator.

\section{CONCLUSION}

We have presented an improved PPV technique for accurate prediction of oscillator phase noise. The proposed FW-PPV technique is generally applicable to predict phase noise for any oscillator circuit, especially for large oscillators with large time constants, on which previous PPV techniques significantly lose accuracy. Simulation results confirm that the FW-PPV is identical to previous PPV techniques when oscillator circuits are small and fast. However, when oscillators are large and with slow time constants, the FW-PPV features much better robustness, predicting the phase noise accurately, while previous PPV techniques lose accuracy significantly and give over-estimated phase noise.

\section{REFERENCES}

[1] A. Demir, A. Mehrotra, and J. Roychowdhury. Phase noise in oscillators: a unifying theory and numerical methods for characterization. IEEE Trans. on Circuits and Systems-I:Fundamental Theory and Applications, 47(5):655-674, May 2000.

[2] X. Lai and J. Roychowdhury. Capturing Oscillator Injection Locking via Nonlinear Phase-Domain Macromodels. IEEE Trans. Microwave Theory Tech., 52(9):2251-2261, September 2004.

[3] X. Lai and J. Roychowdhury. TP-PPV: Piecewise Nonlinear, Time-Shifted Oscillator Macromodel Extraction For Fast, Accurate PLL Simulation. In Proceedings of IEEE ICCAD 2006, November 2006

[4] A. Demir. Phase noise in oscillators: Daes and colored noise sources. In IEEE/ACM International Conference on Computer-Aided Design, November 1998.

[5] A. Demir and J. Roychowdhury. A reliable and efficient procedure for oscillator ppv computation, with phase noise macromodelling applications. IEEE Trans. on Computer-Aided Design of Integrated Circuits and Systems, 22(2):188-197, February 2003.

[6] X. Lai and J. Roychowdhury. A multilevel technique for robust and efficient extraction of phase macromodels of digitally controlled oscillators. In Proc. IEEE Design Automation Conference, pages 1017-1022, July 2006.

[7] T. Mei and J. Roychowdhury. Oscillator-AC: Restoring Rigour to Linearized Small Signal Analysis of Oscillators. In ICCAD 2005, November 2005 .

[8] Y. Saad and M. H. Schultz. Gmres: a generalized minimum residual algorithm for solving non-symmetric linear systems. SIAM Journal on Scientific and Statistical Computing, 7:856-869, September 1986. 